\title{
Accounting for segregation employing granular solid hydrodynamics
}

\author{
Mario Liu ${ }^{1, \star}$ and Yimin Jiang ${ }^{2}$ \\ ${ }^{1}$ Theoretische Physik, Universität Tübingen,72076 Tübingen, Germany \\ ${ }^{2}$ Central South University, Changsha 410083, China
}

\begin{abstract}
Granular solid hydodynamics (GSH) is generalized to account for segregation. The necessary change is minimal, and consists in introducing the concentration $c$ as an additional variable. There is no need for the stress and velocity of every population. GSH's functionality with respect to all other granular phenomena - from elasto-plastic motion and shear band to the $\mu(\mathrm{I})$-rheology - is preserved.
\end{abstract}

Segregation is a granular phenomenon of considerable practical importance. Many researchers have been interested in various aspects of this phenomenon, and have succeeded in setting up theories accounting for them. The descriptions are diverse and possess a high level of sophistication, see [1-3] and references therein. Surprising highlights, for a person entering this area, are especially the description of self-channelizing flows that spontaneously form static levees [4], or the account of grain size dynamics in a five-dimensional space, using a continuous size variable $s$ in addition to time $t$ and space $\vec{r}$ [5].

Though incapable of accounting for segregation, GSH is otherwise a fairly wide-ranged macroscopic theory of granular behavior. The phenomena accounted for include static stress distribution, elasto-plastic motion, and the $\mu(I)$-rheology; also angles of repose and stability, propagation of elastic waves, compaction [6-8]. Furthermore, the theory is derived employing thermodynamics and conservation laws, treating granular media as a thermal, and not-at-all abnormal, system, see [9]. (This last reference contains a minimalist version of GSH that may smoothen one's introduction to it.)

Generalizing GSH to include segregation seems a useful step, as it would clarify the thermodynamic basis of segregation, and would ascertain that the employed equations comply with all constraints. Moreover, this step becomes a necessary one if one aims to account for hybrid phenomena such as motion and segregation in a partially filled rotating cylinder. This is a phenomenon in which especially the following effects and their mutual interaction are relevant: static stress distribution, the $\mu(I)$-rheology, the angles of repose and stability, all in addition to the concentration diffusion. A simple-minded combination of the GSH equations with the advection-diffusion equation is not likely to suffice.

There are two points in the conventional approach that may merit more thoughts and some scrutiny. First, focus- ing on segregation, these theories and equations are tailored to yield a proper account, with less thoughts spent on other granular phenomena such as the ones mentioned above. Yet the ultimate goal of any theory is to eventually arrive at a unified account, similarly broad-ranged as the sum of our observations.

Second, most papers start their description by introducing stress, velocity, and the interaction force $\beta$ for each population of grains, even though distinguishing the stress from forces often lacks uniqueness. Employing scaling laws for the stresses and forces, the given set of differential equations is typically first used to derive an advectiondiffusion equation for the concentration $c$ - usually assuming incompressibility - which is then solved. The scaling law was postulated at first, but then shown to hold through DEM simulations [2]. A classic reference for this type of approach, referred to as mixture theory, is [10].

On the other hand, it is known that the diffusion equation for the concentration $c$ may be derived directly, with no ambiguities, no approximations, and all thermodynamic constraints spelt out, see eg. the well-known textbook by Landau and Lifshitz [11]. For instance, the diffusion equation holds irrespective whether the system is compressible, or whether the scaling laws is valid under all circumstances, say for large soft grains mixed with small hard ones. Also, the derivation reveals the constraint that the diffusion current $J_{k}$ is necessarily proportional to $\nabla_{k}(\chi / \rho)$, where $\rho$ is the total density, see Eq.(1), and $\chi$ is the derivative of the thermodynamic energy $w$ with respect to the concentration $c$, or $\chi \equiv \partial w / \partial c$, that is usually referred to as the chemical potential, see the detailed discussion on the energy $w$ below.

In the following, we shall confine ourselves to a mixture of two types of grains, say large and small, postponing the inclusion of the continuous variable $s$ for a future work. Then we only need to introduce the concentration as a scalar state variable,

$$
c \equiv \varrho^{1} \varrho, \quad \varrho \equiv \varrho^{1}+\varrho^{2}
$$


For $c$ and $\rho-$ as for the rest of the GSH-variables, but unlike the velocity or stress of one grain species - coarsegraining is a simple and unique procedure, carried out for all particles within a given volume element.

For a hydrodynamic theory such as GSH, the most important quantity is the total, conserved, thermodynamic energy density $w+\frac{1}{2} \rho v^{2}$, with $w$ the energy of the local rest frame, and $v_{i}$ the barycentric velocity. For monodisperse grains, the energy of GSH depends on the entropy $s$, granular entropy $s_{g}$, density $\rho$, and the elastic strain $u_{i k}$, or $w=w\left(s, s_{g}, \rho, u_{i j}\right)$. Being the total energy, $w$ contains the energy contributions of all degrees of freedom. The entropy $s$ accounts for the energy of microscopic ones such as electrons and phonons, which is usually referred to as heat. The granular entropy $s_{g}$ accounts for the energy in the mesoscopic, granular degrees of freedom, ie. the quickly fluctuating kinetic and elastic energy of individual grains. One may call this energy contribution, connected to granular jiggling, granular heat. The macroscopic, coarse-grained degrees of freedom, given by the density $\rho$ and the elastic strain $u_{i j}$, are explicitly accounted for, where $u_{i j}$ quantifies the large-scale deformation of the grains. To consider segregation, the dependence on the coarse-grained concentration $c$ needs to be included,

$$
w=w\left(s, s_{g}, \rho, c, u_{i j}\right)
$$

Much physics is encoded in the derivatives of $w$, usually referred to as the conjugate variables,

$$
T \equiv \frac{\partial w}{\partial s}, \quad T_{g} \equiv \frac{\partial w}{\partial s_{g}}, \quad \pi_{i j} \equiv-\frac{\partial w}{\partial u_{i j}}, \quad \chi \equiv \frac{\partial w}{\partial c},
$$

which are, respectively, the temperature, the granular temperature, the elastic stress and the chemical potential. For instance, the total stress $\sigma_{i j}$, defined by $\partial_{t}\left(\rho v_{i}\right)+\nabla_{i} \sigma_{i j}=0$ (with $\partial_{t} \equiv \partial / \partial t$ ), is given as

$$
\sigma_{i j}=P_{T} \delta_{i j}+\pi_{i j}+\rho v_{i} v_{j}-\sigma_{i j}^{D}
$$

where the thermodynamic pressure $P_{T}$ is another derivative,

$$
P_{T} \equiv-\frac{\partial(w / \rho)}{\partial(1 / \rho)},
$$

while $\sigma_{i j}^{D}$ is the dissipative contribution. (For the simplest case, it is the viscous stress.) Similarly, the evolution equation for $c$ is necessarily given as

$$
\begin{array}{r}
\rho\left(\partial_{t}+v_{i} \nabla_{i}\right) c-\nabla_{k} J_{k}=0, \\
J_{k}=D \nabla_{k}(\chi / \rho) .
\end{array}
$$

Only this form is compatible with the conservation laws of energy and the two masses, in addition to the second law of thermodynamics, see the derivation in appendix A. We note that although Eq.(6) is quite similar to the advectiondiffusion equation employed in the literature, the basic idea is somewhat different. The latter has two counteracting terms,

$$
\partial_{t} c+\nabla_{k}\left(c v_{k}\right)-\nabla_{z}\left[S_{r} F(c)\right]=\nabla_{z}\left(D \nabla_{z} c\right),
$$

with the diffusive one $\nabla_{z}\left(D \nabla_{z} c\right)$ homogenizing the concentration, and the advective one $\nabla_{z}\left[S_{r} F(c)\right]$ segregating the two populations. In the stationary case, both terms balance. Eqs. $(6,7)$, on the other hand, have the chemical potential $\chi / \rho$ as a single driving field that both equalize and segregates, stopping to operate only when it is uniform, though $c$ may well be nonuniform then. This is similar to temperature diffusion: The heat current stops when $T$ is uniform, though the entropy $s$ need not be.

More specifically, we have $J_{k}=0$ for $v_{i} \perp \nabla_{i} c$ and a steady state, $\partial_{t} c=0$. (The case $J_{k}=$ const $\neq 0$ is not compatible with the boundary condition $J_{k}=0$.) This implies

$$
\chi / \rho=\text { const. }
$$

Being a derivative of the energy $w$, the chemical potential $\chi$ shares the same variables with $w=w\left(s_{g}, \rho, u_{i j}, c\right)$. (We ignore the entropy $s$ and inhomogeneity of the temperature $T$ here.) Any non-uniformity in the first three variables induces a compensating $c$, to keep $\chi / \rho=$ const. A stationary, non-uniform $c$ is of course segregation.

Moreover, a steady-state $T_{g}$ (which we shall see below is $\sim s_{g}$ ) is proportional to the shear rate, $T_{g} \propto \dot{\gamma}$ : Quantifying granular jiggling, $T_{g}$ relaxes if left alone and is excited by $\dot{\gamma}$, such that $T_{g} \propto \dot{\gamma}$ holds in the steady state. Since flows frequently have non-uniform shear rates and densities, they give rise to segregation.

Note we need to have the diffusion rate $D=D\left(T_{g}\right)$ such, that $D \rightarrow 0$ for grains at rest, $T_{g} \rightarrow 0$. Because whatever the chemical potential then is, concentration distribution is frozen in. Most simply, we may set $D \propto T_{g}$. This implies slow diffusion rates for regions of small $\dot{\gamma}$.

Eqs. $(6,7)$ comprise the structural change of $\mathrm{GSH}$, though we still need to specify the concentration dependence of the energy density $w(c)$, to obtain the chemical potential $\chi \equiv \partial w / \partial c$. Finding the $c$-dependence of the energy is a trial and error process, same as the one we went through to find the energy for mono-disperse systems, an expression that is by now well verified [6-8]. Both are constitutive choices. The energy for mono-disperse grains has the form:

$$
\begin{aligned}
w & =w_{\Delta}+w_{T}, \\
w_{\Delta} & =\sqrt{\Delta}\left[2 \mathcal{B}(\rho) \Delta^{2} / 5+\mathcal{A}(\rho) u_{s}^{2}\right], \\
\Delta & \equiv-u_{k k}, u_{s}^{2} \equiv u_{i j}^{*} u_{i j}^{*}, u_{i j}^{*} \equiv u_{i j}+\Delta \delta_{i j} / 3, \\
w_{T} & =s_{g}^{2} /(2 \rho b)=\rho b T_{g}^{2} / 2, \\
T_{g} & \equiv \partial w / \partial s_{g}=s_{g} / \rho b, \\
b & =b_{1} / \rho+b_{0}\left[1-\rho / \rho_{c p}\right]^{a}, \quad a \approx 0.1 .
\end{aligned}
$$

The contribution $w_{\Delta}$ is the elastic energy, a function of the elastic strain $u_{i j}$, which quantifies the coarse-grained deformation of the grains. The term $w_{T}$ is the energy of granular jiggling, the quickly fluctuating kinetic and elastic energy of the grains. (Note $T_{g}^{2} \propto T_{k}$, where $T_{k}$ is the temperature used in the kinetic theory.) With the expressions $\mathcal{B}(\rho), \mathcal{A}(\rho)$ given in $[6,7], w_{\Delta}$ and the elastic stress $\pi_{i j}$ have proven suitable for the account of various static stress distributions, propagation of elastic waves and elasto-plastic 
motion [8]. $w_{T}$ and

$$
P_{T} \equiv-\frac{\partial(w / \rho)}{\partial(1 / \rho)}=-\frac{\partial\left(w_{T} / \rho\right)}{\partial(1 / \rho)}
$$

from granular jiggling are also well-verified. (The contribution from $w_{\Delta}$ is of higher order and may be neglected.) For $\rho \rightarrow 0$, the first term in $b$ dominates, $b \approx b_{1} / \rho$, then $P_{T}$ reduces to that found in kinetic theories. (There is then no elastic stress, $\pi_{i j}=0$.) For densities closer to the closepacked value $\rho_{c p}$, the term $\propto b_{0}$ dominates, accounting for compaction and the $\mu(I)$-rheology.

If $w$ is to depend on $c$, we need to specify its dependence such that it reverts to the above formulas for $c=0$ and $c=1$. Furthermore, thermodynamic stability or the convexity of energy requires

$$
\frac{\partial^{2} w}{\partial c^{2}}>0, \quad \frac{\partial^{2} w}{\partial c^{2}} \frac{\partial^{2} w}{\partial \rho^{2}}-\frac{\partial^{2} w}{\partial \rho \partial c}>0
$$

However, a partially concave $w$ does exist in nature, eg. in mixtures of liquids. It then implies that certain concentration values cannot be realized in this mixture. The system will demix, with a region of high concentration, $c=c_{1}$, another of low concentration, $c=c_{2}$, such that $w\left(c_{1}\right)$ and $w\left(c_{2}\right)$ are convex. Analogously, a partially concave $w$ for a mixture of two types of grains does not have to be illchosen. It may appropriately reflect the observation that certain c-values is not realized in equilibrium. The system will then also demix, with a region of say only large grains, another with a $c$ value possessing a stable, convex energy.

In specifying the c-dependence, we shall for two reasons only modify $w_{T}$, not $w_{\Delta}$. First, the fluctuating part of the kinetic stress was identified as the dominant cause for segregation, see [12], and it is closely related to $P_{T} \propto T_{g}^{2}$.

Second, the elastic contribution of $w_{\Delta}(c)$ to the chemical potential is typically small, $\chi_{\Delta} \ll \chi_{T}$, hence $\chi=$ $\chi_{\Delta}+\chi_{T} \approx \chi_{T}$. The reason is, in the elastic pressure, $P_{\Delta} \equiv \frac{1}{3} \pi_{k k} \propto \mathcal{B} \Delta^{1.5}$, with typically $\Delta \lesssim 10^{-3}$, a large $\mathcal{B}$ compensates a small $\Delta^{1.5}$. Now, since $\chi_{T}$ is of the order of $P_{T} / c$, and $\chi_{\Delta} \propto(\mathcal{B} / c) \Delta^{2.5} \propto \Delta P_{\Delta}$, we have $\chi_{T} \gg \chi_{\Delta}$, if $P_{\Delta}$ is comparable to $P_{T}$. (For $T_{g}^{2}$ sufficiently small, we have $P_{T} \propto T_{g}^{2} \ll P_{\Delta}$. But this regime is probably irrelevant for segregation.)

Modifying $w_{T}$, one easy way to proceed is to replace its constants, especially

$$
\rho_{c p}(c)=\rho_{c p}^{0}+h c(1-c)
$$

because we know that $\rho_{c p}$ changes in the mixture, with $h$ positive and growing with the ratio of the two radia, because the small grains fill the void between the large ones. If we take $h$ depending on $c$, say linearly, $\rho_{c p}(c)$ is not symmetric with respect to $c \rightarrow 1-c$, and its maximum is not at $c=\frac{1}{2}$. If $\rho_{c p}^{0}$ is different for $c=1$ and $c=0$, it is to be replaced by

$$
\rho_{c p}^{0} \rightarrow c \rho_{c p}^{1}+(1-c) \rho_{c p}^{2}
$$

If Eq.(13) is the only change, we have

$$
\begin{gathered}
\chi \approx \partial w_{T} /\left.\partial c\right|_{s_{g}}=-\left(\rho T_{g}^{2} / 2\right) \partial b / \partial c, \\
\frac{\partial b}{\partial c}=\frac{b_{0} a \rho}{\rho_{c p}^{2}}\left(1-\frac{\rho}{\rho_{c p}}\right)^{a-1} \frac{\partial \rho_{c p}}{\partial c}, \\
\partial \rho_{c p} / \partial c=h(1-2 c)+c(1-c) \partial h / \partial c .
\end{gathered}
$$

Note $\partial^{2} w / \partial c^{2}>0$ for $h=$ const, though it becomes negative for $c<1 / 3$ if $h \propto c$. If $h=$ const, we have

$$
\frac{\chi}{\rho}=-\frac{T_{g}^{2} h[1-2 c] b_{0} a \rho\left[2 \rho_{c p}^{2}(c)\right]^{-1}}{\left[1-\rho / \rho_{c p}(c)\right]^{1-a}}
$$

Since the $c$-dependence of the denominator varies more weakly, we may frequently neglect it, replacing $\rho_{c p}(c)$ with $\rho_{c p}^{0}$. Note that the structure of this formula,

$$
\chi / \rho=-T_{g}^{2} h[1-2 c] f(\rho),
$$

is retained [with a modified $f(\rho)$ ], if Eq.(13) works also for replacing $b_{0}$ alone, though replacing $b_{0}$ and $\rho_{c p}$ simultaneously is certainly more realistic.

Replacing $b_{1}$ does not lead to segregation: For a rarefied granular gas, we have $\frac{1}{2} b_{1} T_{g}^{2}=\frac{3}{2}(\rho / M) k_{B} T$, and if bi-disperse $\frac{1}{2} b_{1} T_{g}^{2}=\frac{3}{2} \rho k_{B} T\left[c / M_{1}+(1-c) / M_{2}\right]$. This implies the substitution

$$
b_{1} \rightarrow b_{1}^{0}\left[c+(1-c)\left(M_{1} / M_{2}\right)\right]
$$

Being linear in $c$, this form does not lead to a $c$-dependence of the chemical potential $\chi$.

A robust conclusion is, flows with a constant $\dot{\gamma}$ and a constant density do not give rise to segregation.

\section{References}

[1] J.M.N.T. Gray and A.R. Thorton Proc. R. Soc. A 461, 1447 (2005).

[2] D. R. Tunuguntla, O. Bokhove, and A. R. Thornton, Journal of Fluid Mechanics 749, 99 (2014).

[3] P. Gajjar, K. van der Vaart, A. R. Thornton, C. G. Johnson, C. Ancey and J. M. N. T. Gray. J. Fluid Mech., vol. 794, pp. 460-505 (2016)

[4] S. Deboeuf, E. Lajeunesse, O. Dauchot, B. Andreotti. Phys.Rev.Lett. 97, 158303 (2006)

[5] B. Marks, P. Rognon, and I. Einav, Journal of Fluid Mechanics 690, 499 (2012).

[6] Y.M. Jiang, M. Liu. Granular Matter, 11:139(2009), free download: www.springerlink.com/ content/a8016874j8868u8r/fulltext

[7] Y.M. Jiang and M. Liu. Acta Mech. 225, 2363-2384 (2014)

[8] Y.M. Jiang and M. Liu. Applying GSH to a wide range of experiments in granular media. Eur. Phys. J. E (2015) 38:15

[9] Y.M. Jiang and M. Liu. Why granular media are thermal, and quite normal, after all. Eur. Phys. J. E (2017) 40: 10 
[10] L.W.Morland, Flow of viscous fluid through a porous deformable matrix Surveys in Geophysics (1992) 13:209 268.

[11] L. D. Landau and E. M. Lifshitz. Fluid Mechanics. Butterworth-Heinemann (1987).

[12] K.M. Hill and D.S. Tan. Segregation in dense sheared flows: gravity, temperature gradients, and stress partit. J. Fluid Mech., 756, 54-88. (2014)

\section{A Deriving the c-equation}

Starting with the equations for $\rho^{1}, \rho^{2}$,

$$
\begin{aligned}
& \partial_{t} \rho^{1}+\nabla_{k}\left(\rho^{1} v_{k}^{1}\right)=0, \\
& \partial_{t} \rho^{2}+\nabla_{k}\left(\rho^{2} v_{k}^{2}\right)=0,
\end{aligned}
$$

we rewrite them as

$$
\begin{array}{r}
\partial_{t} \rho^{1}+\nabla_{k}\left(\rho^{1} v_{k}\right)-\nabla_{k} J_{k}=0, \\
\partial_{t} \rho^{2}+\nabla_{k}\left(\rho^{2} v_{k}\right)+\nabla_{k} J_{k}=0, \\
\text { where } \quad J_{k}=\rho^{1} \rho^{2}\left(v_{k}^{2}-v_{k}^{1}\right) / \rho, \\
v_{k}=\left(\rho^{1} v_{k}^{1}+\rho^{2} v_{k}^{2}\right) / \rho .
\end{array}
$$

Combining both equations, we obtain

$$
\begin{aligned}
\partial_{t} \rho+\nabla_{k}\left(\rho v_{k}\right) & =0, \\
\rho\left(\partial_{t} c+v_{k} \nabla_{k} c\right)-\nabla_{k} J_{k} & =0,
\end{aligned}
$$

which is the continuity equation and the equation for $c \equiv$ $\rho^{1} / \rho$ of the form given in Eq.(2).

Next, we derive the expression for $J_{k}$, in terms of the variables already present, as Eq.(20), containing $v_{k}^{1}, v_{k}^{2}$, is not useful. For simplicity, we shall go to the rest frame, $v_{k} \equiv 0$, because the same consideration in a general frame is rather more complicated, and produces results we are not interested in here.

As the conserved energy density $w=w\left(s, \rho^{1}, \rho^{2}\right)$ is a function of the entropy density $s$, in addition to $\rho^{1}, \rho^{2}$, we write

$$
\mathrm{d} w=T \mathrm{~d} s+\mu^{1} \mathrm{~d} \rho^{1}+\mu^{2} \mathrm{~d} \rho^{2},
$$

where $T, \mu^{1}, \mu^{2}$ are the temperature and the respective chemical potentials. (The dependence on the granular entropy $s_{g}$ and the elastic strain $u_{i j}$ is also neglected here for simplicity.) We rewrite this relation as

$$
\begin{gathered}
\mathrm{d} w=T \mathrm{~d} s+\mu^{2} \mathrm{~d} \rho+\left(\mu^{1}-\mu^{2}\right) \mathrm{d} \rho^{1}, \\
=T \mathrm{~d} s+\mu \mathrm{d} \rho+\chi \mathrm{d} c, \quad \text { with } \\
\chi=\rho\left(\mu^{1}-\mu^{2}\right), \mu=(1-c) \mu^{2}+c \mu^{1} .
\end{gathered}
$$

Taking the temporal derivative of Eq.(24), inserting the conservation equations (for the rest frame): $\partial_{t} w=\nabla_{k} Q_{k}$, $\partial_{t} \rho=0, \partial_{t} \rho^{1}=\nabla_{k} J_{k}$, and the balance equation $T \partial_{t} s=$ $R>0$, we find

$$
\begin{aligned}
& \nabla_{k} Q_{k}-R=\left(\mu^{1}-\mu^{2}\right) \nabla_{k} J_{k} \\
& \quad=\nabla_{k}\left[\left(\mu^{1}-\mu^{2}\right) J_{k}\right]-J_{k} \nabla_{k}\left(\mu^{1}-\mu^{2}\right) .
\end{aligned}
$$

This equation is an identity that has to be satisfied at all time and in all of space. Taking $Q_{k}=\left(\mu^{1}-\mu^{2}\right) J_{k}$, and Eq.(3),

$$
J_{k}=D \nabla_{k}\left(\mu^{1}-\mu^{2}\right)=D \nabla_{k}(\chi / \rho),
$$

with $D>0$, satisfies energy conservation and ensures that the entropy production $R$ is positive-definite. 